ARTICLE

https://doi.org/10.1038/s41467-019-13511-9

\title{
A transferable machine-learning framework linking interstice distribution and plastic heterogeneity in metallic glasses
}

Qi Wang ${ }^{1 \star} \&$ Anubhav Jain (10) ${ }^{1 \star}$

When metallic glasses (MGs) are subjected to mechanical loads, the plastic response of atoms is non-uniform. However, the extent and manner in which atomic environment signatures present in the undeformed structure determine this plastic heterogeneity remain elusive. Here, we demonstrate that novel site environment features that characterize interstice distributions around atoms combined with machine learning $(M L)$ can reliably identify plastic sites in several $\mathrm{Cu}-\mathrm{Zr}$ compositions. Using only quenched structural information as input, the ML-based plastic probability estimates ("quench-in softness" metric) can identify plastic sites that could activate at high strains, losing predictive power only upon the formation of shear bands. Moreover, we reveal that a quench-in softness model trained on a single composition and quench rate substantially improves upon previous models in generalizing to different compositions and completely different $M G$ systems $\left(\mathrm{Ni}_{62} \mathrm{Nb}_{38}, \mathrm{Al}_{90} \mathrm{Sm}_{10}\right.$ and $\mathrm{Fe}_{80} \mathrm{P}_{20}$ ). Our work presents a general, data-centric framework that could potentially be used to address the structural origin of any site-specific property in MGs.

\footnotetext{
${ }^{1}$ Lawrence Berkeley National Laboratory, Energy Technologies Area, 1 Cyclotron Road, Berkeley, CA 94720, USA. *email: qiwang.mse@gmail.com; ajain@lbl.gov
} 
U pon sufficiently rapid cooling, many metallic melts become frozen and form amorphous alloys or metallic glasses $(\mathrm{MGs})^{1-5}$. The combination of metal and glass not only produces many technologically useful properties but also introduces intriguing and incompletely understood behaviors ${ }^{6-8}$. Understanding and controlling deformation is one of the greatest challenges in $\mathrm{MGs}^{9-15}$. Unlike in crystals, each atom in a disordered material has a unique atomic environment, and as a result, when subjected to mechanical stimuli, their response can, in principle, be different. Such heterogeneity makes it notoriously difficult to establish a causal link between structure and deformation $^{9-11,14,15}$.

Several signatures have previously been proposed to characterize the local glass structure and serve as indicators of plastic heterogeneity, such as soft modes ${ }^{16-18}$, local yielding stress ${ }^{19}$, local thermal energy ${ }^{20}$, vibrational mean-squared displacement $^{21,22}$, and flexibility volume ${ }^{23}$. These indicators are based on the measurement of physical observables and have clear interpretations, yet typically require detailed knowledge of atomistic interactions. Attempts from a purely structural perspective (i.e., with knowledge of only the atomic positions) have long been frustrated owing to the lack of representations to sufficiently encode the structural heterogeneity. Recently, researchers have made notable progress by combining symmetry functions as structural representations with machine learning (ML) to establish predictive models for the plasticity and dynamics of various disordered solids and liquids ${ }^{24-27}$.

The use of symmetry functions (originally proposed to fit $\mathrm{ML}$ interatomic potentials ${ }^{28,29}$ ) to establish structure-property relationships in MGs has both advantages and drawbacks. A major advantage is that they can be considered as quite complete and can successfully distinguish many different types of environments ${ }^{24-27}$. However, the complex and less intuitive transformations, especially for the angular functions, makes it more challenging to interpret the ML models and extract scientific insights from them. In addition, to our best knowledge no study has demonstrated how ML models employing symmetry functions generalize to different compositions and different chemical systems (i.e., without re-training). As we will later demonstrate, models trained on symmetry functions may be system specific and therefore limited in their ability to establish more general structure-plasticity mappings that hold across compositions and chemistries.

In this work, we develop a new structural representation by extracting features from the interstice distributions in short and medium range that are conceptually related to local susceptibility to rearrangement (Fig. 1). We find that this representation has advantages in interpretability and generalizability over symmetry functions ${ }^{24-26}$ as well as conventional signatures ${ }^{30-38}$ (e.g., coordination number $(\mathrm{CN})^{30}$, Voronoi indices ${ }^{30}$, characteristic motifs $^{31,32}$, volume metrics ${ }^{34,35}$, and $i$-fold symmetry indices $\left.{ }^{36}\right)$. We use these features to explore how the atomic features present in the undeformed, quenched configuration affects plasticity even at large strains and long time scales (see Supplementary Fig. 1 for an illustration of differences from previous works). The plastic probability estimates of the ML model, which we call "quench-in softness (QS)," serves as an indicator of the defective nature of site environments and enables us to survey the landscape of soft and hard packings within MGs. Remarkably, we demonstrate that a QS metric trained on one MG is generalizable across compositions, quenching conditions, and even different chemical systems, suggesting that the traits of atom sites prone to rearrangement could be consistent across different MGs (especially ones containing only metallic elements). Furthermore, the ML framework is general and can be conceivably applied to predict any site-specific property of MGs.

\section{Results}

Interstice distribution in the short and medium range. To establish a ML link between the site environments and plastic heterogeneity, we first represent the site environments such that they capture the structural heterogeneity in MGs.

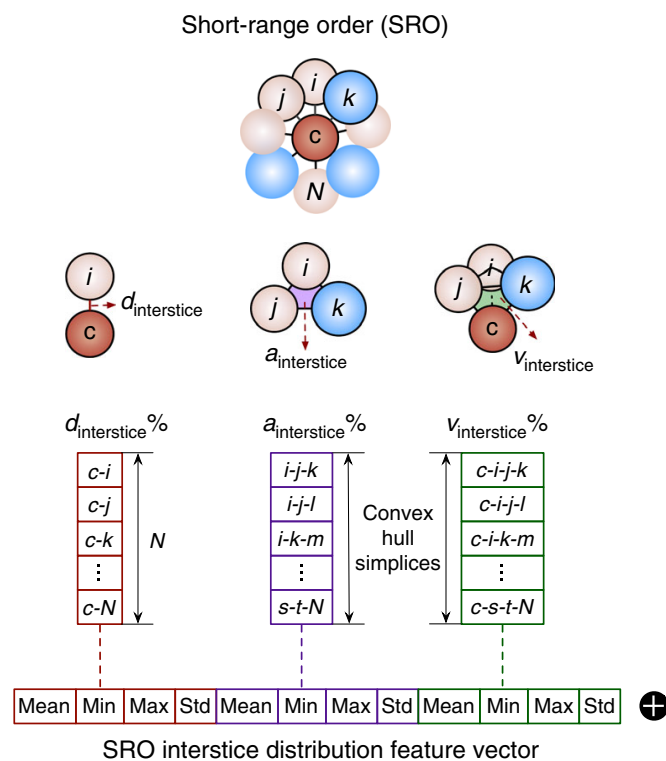

SRO interstice distribution feature vector
Medium-range order (MRO)
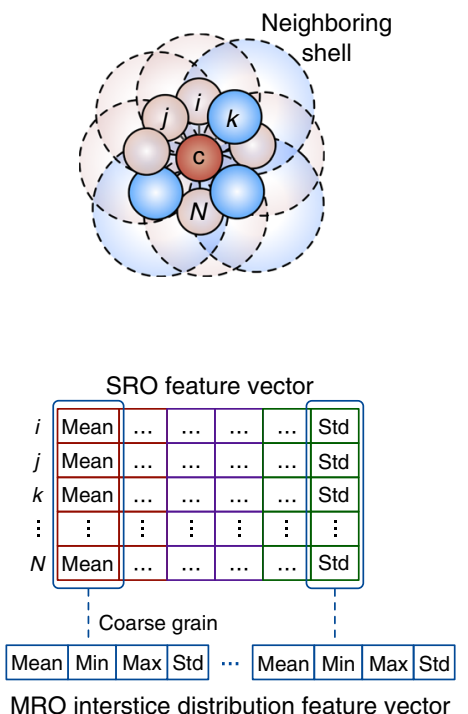

Fig. 1 Representing site-specific interstice distribution in the short and medium range. We define the distance, area, and volume interstice (in ratio) as the fraction of neighboring distances, triangulated surface area, and tetrahedra that are not covered by atom spheres. We characterize the distance, area, and volume interstices across all nearest-neighbor bonds and convex hull simplices and take statistics (mean, min, max, and std) of them to describe the anisotropy of interstice distribution in the short-range order (SRO) around each atom. The SRO feature vectors of all neighbors can be reduced to a single medium-range order (MRO) feature vector by calculating statistics across neighbors (mean, min, max, and std). The SRO and MRO features are then concatenated $(\oplus)$ as a representation of the interstice distributions around each atom. The features are then served as input to a ML algorithm (gradient boosting decision tree in this work) to train and predict the heterogeneous plastic response of atoms in MGs. 
It is well established that interstices in crystals (also called holes or voids) strongly influence diffusion, deformability, and other transport properties ${ }^{39}$. In MGs, however, owing to the disordered structure, the interstices are more difficult to define and characterize 6 . In previous studies, Yang et al. have proposed that the atomic packing efficiency, the ratio between the volume of embedded atoms and the total volume of the cluster (equivalent to " 1 -interstice fraction"), is strongly correlated with glass-forming ability of $\mathrm{MGs}^{34}$. In terms of plastic deformation, as the rearrangements can be directional and anisotropic, an average interstice fraction alone may be insufficient to distinguish the plastic sites. Indeed, as we will later demonstrate, a more complete representation of interstice distribution, as well as extensions beyond short-range order (SRO) to medium-range order (MRO), are required to obtain more accurate models.

We first characterize the distance, area, and tetrahedral volume interstices in the neighboring cluster to construct the SRO descriptors (Fig. 1). Each of these metrics is a measure of the relative amount of empty space around each atom as determined using atomic sphere models. The distance interstice is the fraction of a bonding line unoccupied by the atom spheres; it can be negative if the atom spheres overlap. The area interstice is the unoccupied area within a triangulated surface formed by atom triplets in the convex hull formed by neighbors, and the volume interstice is the unoccupied portion of a tetrahedra formed between the central atom and neighbor atom triplets; these metrics are typically non-negative. Specifically, we determine neighbors using Voronoi tessellation analysis (an exception is noted later), with small facets with areas $<5 \%$ of the average facet areas removed. To calculate the area and volume interstices, we then derive the convex hull ${ }^{40,41}$, composed by triangulated facets, of the Voronoi neighbors. We calculate the atom-packed area and volume in each triangulated surface and tetrahedron by adding up the circular sector area and cone volumes at each vertex (through calculating the triangular angles and solid angles), and then subtract the atom-packed area and volume from the entire triangulated facet area and tetrahedron volume to calculate the interstices (Fig. 1; see Methods for details).

Iterating the above procedure for all possible interstices in the neighboring environment of each atom will generate three vectors whose length is the number of neighbor atoms (distance interstice) or the number of convex hull simplices (area or volume interstice). In essence, the coordination environment in MGs is anisotropic, which can be reflected in the inequality of the distance, area, and volume interstice vector elements. To describe this anisotropy, we derive statistics (mean, min, max, and standard deviation) of the interstice vector elements to featurize the interstice distribution around an atom (Fig. 1). Other methods can be grouping the interstice vector elements into histogram grids of fixed bins and the features then become a vector of all the values of these histograms (Gaussian smearing can used as an option to reduce noise of discrete histogram values and obtain a smoothed distribution). In addition to using Voronoi tessellation to determine the neighbors, the distance interstice metrics can be easily augmented by those calculated from neighbors within a cutoff distance (e.g. $4.0 \AA$ for $\mathrm{Cu}-\mathrm{Zr}$ MGs), and if so, the number of SRO features will increase from 12 to 16 .

We next represent the interstice distributions in MRO (Fig. 1). Although MRO has long been proposed to be vital to determining glass properties, few MRO signatures are available in literature ${ }^{42-44}$. Here we generalize a coarse-graining strategy to use the statistics of SRO features of an atom's neighbors to describe the center atom itself. Specifically, we process an SRO feature $F^{S R O}$ to calculate its statistics across the neighbors of atom $i$, and the MRO features will be

$$
F_{i}^{\mathrm{MRO}}=\operatorname{Stats}\left(F_{1}^{\mathrm{SRO}}, F_{2}^{\mathrm{SRO}}, \ldots, F_{n}^{\mathrm{SRO}}\right), n \in N(i)
$$

where $n$ iterates the neighbors $N(i)$ determined by Voronoi tessellation analysis or within a cutoff distance and Stats represent the summary statistics of mean, min, max, and standard deviation. This allows for automatic encoding of the second neighbor effect and is similar to the idea of imposing convolution ${ }^{45}$ over neighbors for longer-scale feature extraction. Crucially, this strategy allows us to transform any numeric SRO feature into a set of MRO features. Overall, we see that the SRO and MRO features have clear physical meanings in describing the interstices around each atom and are robust to varying scales of atomic sizes due to being in the form of ratio.

To summarize, we establish a representation to describe the heterogeneous interstice distribution that spans SRO and MRO around atoms in MGs. Each atom is represented by 80 variables, concatenated from 16 SRO and 64 MRO features $\left(F=F^{S R O} \oplus\right.$ $\left.F^{\mathrm{MRO}}\right)$. To reduce model complexity and improve interpretability, we further remove highly correlated features and use recursive feature elimination (Supplementary Fig. 5) to reduce the representation to 15 features (listed in Supplementary Table 2, in descending order of the five fold cross-validation (CV) averaged feature importances).

The codes for this representation, together with many existing features (such as Voronoi indices, volume metrics, $i$-fold symmetry, bond-orientational order, and symmetry functions), are publicly available in amlearn (https:/github.com/Qi-max/ amlearn), our package targeted for ML in amorphous materials, and matminer ${ }^{46}$ (https://github.com/hackingmaterials/matminer). In amlearn, we wrap Fortran 90 subroutines and functions with Python using $\mathrm{f} 2 \mathrm{py}^{47}$ to combine usability and fast computation. This representation is general and can potentially describe the site environments of any MG. We will later show that this representation improves upon the predictive ability of recognized signatures and can even be highly generalizable between different compositions and chemical systems.

Mapping plastic atoms to quenched-in defects. Following the feature extraction or fingerprinting step, we train a ML model to map the features to the property of interest. In our case, this is whether an atom in the quenched structure is susceptible to plastic rearrangement or not. In this work, we select $\mathrm{Cu}_{x} \mathrm{Zr}_{1-x}$ ( $x=50,65$, and 80 at.\%), which are promising binary MG formers ${ }^{42-44,48-51}$, as principal alloys and extend analyses to $\mathrm{Ni}_{62} \mathrm{Nb}_{38} 52,53, \mathrm{Al}_{90} \mathrm{Sm}_{10} 54$, and $\mathrm{Fe}_{80} \mathrm{P}_{20}{ }^{55} \mathrm{MGs}$. To generate data for ML, we quench large glass samples $(345,600$ atoms for $\mathrm{Cu}-\mathrm{Zr}$ and 131,072 for other MGs) under quenching rates of $5 \times 10^{10}$, $5 \times 10^{11}$, or $5 \times 10^{12} \mathrm{~K} \mathrm{~s}^{-1}$ and apply uniaxial compressive or tensile strain under strain rates of $2.5 \times 10^{7}$ or $1 \times 10^{8} \mathrm{~s}^{-1}$ at $50 \mathrm{~K}$, with periodic boundary conditions along $X$ and $Z$ or along all three directions, using molecular dynamics simulations (see Methods and Supplementary Fig. 2). Here we use gradientboosted decision tree (GBDT) as the ML algorithm, which builds the prediction model in an iterative manner to construct an ensezmble of decision tree learners through boosting ${ }^{56}$. To rigorously test the ML models, we quench and compress three independent samples for each combination of composition and quenching rate and use two of the three samples per condition for training, whereas the third sample is set aside for generalization tests and completely unseen during model development. Owing to the imbalanced nature of the datasets, we use equal undersampling for the training data to create a balanced dataset. We then use fivefold CV to train the GBDT models that use the feature vectors of the undeformed configuration to classify atoms that deform 
plastically up to a strain of $4.0 \%$ (see Methods for details). The trained GBDT models are then tested on the completely set-aside generalization sample (thus excluding any trivial information leakage from training) without any undersampling. As a measure of plastic deformation, we use accumulative non-affine displacement ${ }^{10}\left(D^{2}\right)$ at a relatively large strain $(4.0 \%)$ with reference to the undeformed configuration and set a threshold value of $5.0 \AA^{2}$ to distinguish the plastic and non-plastic atoms. We compare $D^{2}$ with other plastic indicators in Supplementary Fig. 4. The models are compared using their area under receiver operating characteristic curve (AUC-ROC) score (see Methods for the motivation) as well as recall (for consistency with previous works ${ }^{24-26}$ ).

We begin by discussing $\mathrm{Cu}_{65} \mathrm{Zr}_{35}$ MG quenched under a rate of $5 \times 10^{10} \mathrm{~K} \mathrm{~s}^{-1}$ as an example (other MGs are discussed later). $\mathrm{Cu}_{65} \mathrm{Zr}_{35}$ is known as an optimum glass former in the $\mathrm{Cu}-\mathrm{Zr}$ system $^{42-44,48-50}$. For the ML task of using only the undeformed configuration to predict the plastic atoms accumulated up to a relatively large strain of $4.0 \%$, the AUC-ROC on a set-aside test glass configuration is 0.771 , and the ML model captures $74.2 \%$ of the true plastic rearrangements (recall). We compare this against baseline models (random, most frequent, and minority predictors) and they give AUC-ROCs of roughly 0.50 (random) or strictly 0.50 (most frequent and minority), suggesting that such prediction is non-trivial to achieve.

We next test whether our model can be improved by adding conventional structural features for MGs. We characterize the atoms with another seven sets of existing geometrical SRO features $\left(\mathrm{CN}^{30}\right.$, Voronoi indices ${ }^{30}$, characteristic motifs ${ }^{31,32}$, volume metrics ${ }^{34,35}, i$-fold symmetry indices ${ }^{36}$ and their weighted version, bond-orientational order ${ }^{33}$ ) and one chemical SRO feature set (numbers of each element type in the neighboring shell, Warren-Cowley parameters ${ }^{37,38}$ ), totaling 49 SRO features (see Methods), and the AUC does not increase despite the increased number of features (Supplementary Table 5). We also follow the coarse-graining method described above to further generate $10 \mathrm{MRO}$ feature sets, totaling $209 \mathrm{MRO}$ features (Methods), and the AUC has a negligible increase of $\sim 0.001$ (Supplementary Table 7). We also test against 166 symmetry functions following the parameters of previous works ${ }^{24-26}$ and train a GBDT model with exactly the same data and CV splits (see Methods section). The resulting AUC is 0.751 (Supplementary Table 3), which is slightly lower than but comparable to our result. Nonetheless, we will later show that, as the formulation of symmetry function is sensitive to length scales, their generalizability to different compositions would be

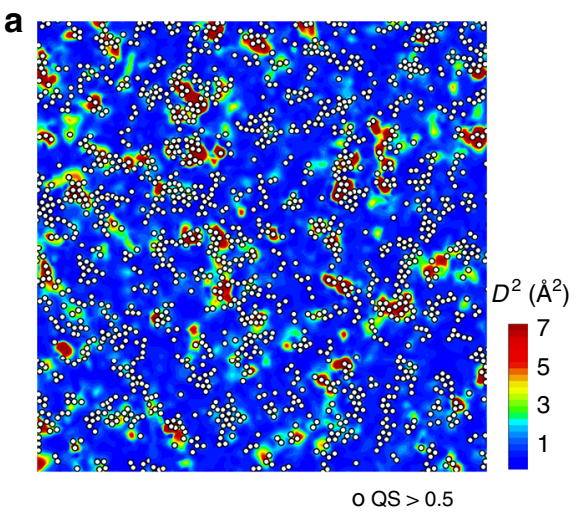

restricted, while our representation and trained ML models exhibit superior generalizability to different compositions and even different chemical systems without re-training the model.

Along with classification, GBDT can evaluate the probability of each atom to be plastic, which can be considered as an indicator of the plastic susceptibility. For example, a probability of 0.50 indicates the model predicts the atom to have an equal probability to be plastic or non-plastic, and the larger the probability, the greater the likelihood for the atom to be plastic. This is similar to the previously introduced idea of "softness" (distance from the support vector machine (SVM) hyperplane) ${ }^{25,26}$ but provide (i) well-calibrated probability estimates bounded in the range $[0,1]$ that can serve as confidence level of classification and does not need further calibration to transform the unbounded SVM distances into probabilities (Supplementary Fig. 7) and (ii) determined from the undeformed, quenched configuration immediately after quenching and thus could be considered as softness that is quenched-in during glass transition, i.e., QS.

Figure $2 \mathrm{a}$ visualizes the atoms with $\mathrm{QS}>0.5$, versus the contour map of $D^{2}$ distribution of the set-aside glass configuration at the strain of $4.0 \%$. Notably, plastic rearrangements have a high propensity to originate from the regions with large QS. The distribution of QS also captures some clustering tendency of plastic atoms, due to the enhanced length scale by incorporating features beyond SRO. Figure $2 \mathrm{~b}$ shows the likelihood that an atom with an observed value of $D^{2}$ is predicted to be plastic by ML when given the undeformed configuration as input. This possibility increases with $D^{2}$, indicating that the more plastic an atom is after applying strain, the more likely it is to be predicted as plastic by the ML model using the initial structure.

The ability to reasonably predict plastic atoms at large strains using the undeformed configuration itself suggests the existence of a long-lived inheritance of plastic heterogeneity on the quenched structure. Here our prediction horizon (strain $4.0 \%$, or equivalently $1.6 \mathrm{~ns}$ ) from a single structural snapshot is much longer than the previous ML framework (for example, strain $0.02 \%$, or equivalently 400 timesteps $^{24}$ ). Our model is unique in that, once trained successfully, only a single undeformed snapshot is needed to predict plastic atoms even at relatively large strains. Different from collecting stepwise snapshots to construct the datasets, here our dataset only samples the quenched atomic environment of each atom once (Supplementary Fig. 1), and the model is further evaluated with an external test glass configuration that undergoes independent quenching and deformation and

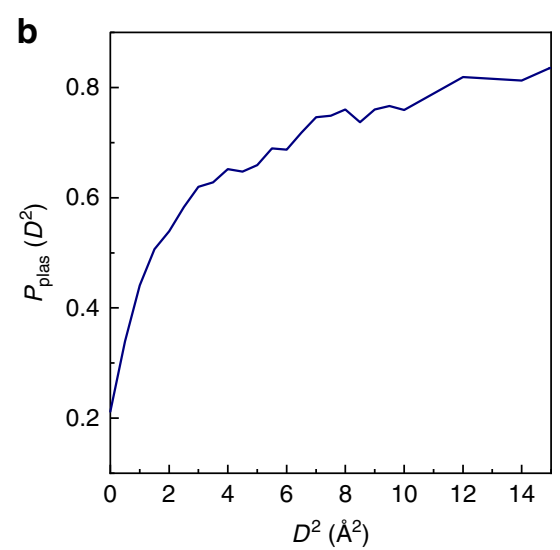

Fig. 2 Predicting plastic atoms using interstice features and machine learning. a The predicted plastic atoms (white circles) by our ML model versus the distribution of $D^{2}$ from atomistic simulations in a slice of $2.0 \AA$ thickness at a strain of $4.0 \%$ of the prototypical glass of $C u_{65} Z_{35}$ quenched at a rate of $5 \times$ $10^{10} \mathrm{~K} \mathrm{~s}^{-1}$. All atoms predicted with quench-in softness (QS) $>0.5$ are marked as white circles. $\mathbf{b}$ The fraction of atoms predicted as plastic (QS $>0.5$ ) by our ML model (using the undeformed configuration alone as input) as a function of the actual $D^{2}$ value at a strain of $4.0 \%$. 
has different initial configuration and deformation process with the trained configurations.

Spectrum of QS in MGs. To understand the variety of site environments present within a MG, we examine the distribution of QS (Fig. 3a). A long tail in the higher QS (soft) end is observed, while the low QS (hard) end distributes more smoothly. We further plot the probability that an atom rearranges as a function of $\mathrm{QS}^{26}$ (Fig. 3b). This probability is a strong function of QS, increasing by several orders of magnitude from the hardest to the softest atoms. Furthermore, a value of $\mathrm{QS}=0.5$ corresponds to a plastic likelihood that is equal to the overall fraction of plastic atoms. This is demonstrated in the right-side axis of Fig. 3b, where the quantity $P$ (plastic|QS) $/ P$ (plastic) is close to 1.0 for $\mathrm{QS}=0.5$. In the lower QS (hard) end, the curve bends at $\sim 0.1$, below which the atoms are at least $\sim 10$ times less probable to be plastic than average. These atoms cover $\sim 13 \%$ of the total atoms and could be viewed as the hardest or most solid-like atoms. Their average $D^{2}$ is $\sim 0.55 \AA^{2}$, suggesting that they mostly respond elastically with minor non-affine rearrangement. In the soft end, we consider atoms with QS $>0.7$ (the beginning of the soft tail) as the softest, or most liquid-like atoms, with a similar atomic fraction of $\sim 11 \%$.

We proceed to address how these characteristic atoms pack in space. We first perform fractal dimensionality sampling ${ }^{57,58}$ for the hardest and softest atoms using the power-law scaling of the mass distribution $M(r) \sim r^{D}$, where $M(r)$ denotes the number of atoms of each type within radius $r$ centered by an atom (Fig. 3c). Theoretically, the slope $D$ of the $M(r)$ curve in log-log plot is the dimensionality, and $D<3$ indicates fractal structure ${ }^{57,58}$ (i.e., the number of atoms does not straightforwardly increase with the volume of an enclosing sphere). We see the hardest and soft atoms both show fractal-like packing in length scales $<10 \AA(\sim 4$ neighboring shells), beyond which the packing becomes more space filling ( $D$ close to 3 ). The fractal-like characteristics is much stronger in the hardest atoms than the softest ones. We further extract the pair correlation functions $g(r)$ of the two groups of atoms (Fig. $3 \mathrm{c}$ inset). The first peak of the hardest atoms is higher, suggesting a higher neighboring tendency. Beyond the neighboring shell, the hardest atoms still exhibit clear coordination peaks up to $\sim 4$ neighboring shells, while the peaks of softest atoms quickly smear out. The distinct coordination behaviors suggest a

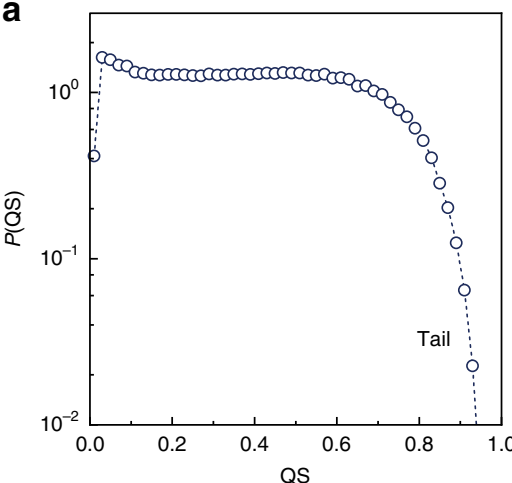

d

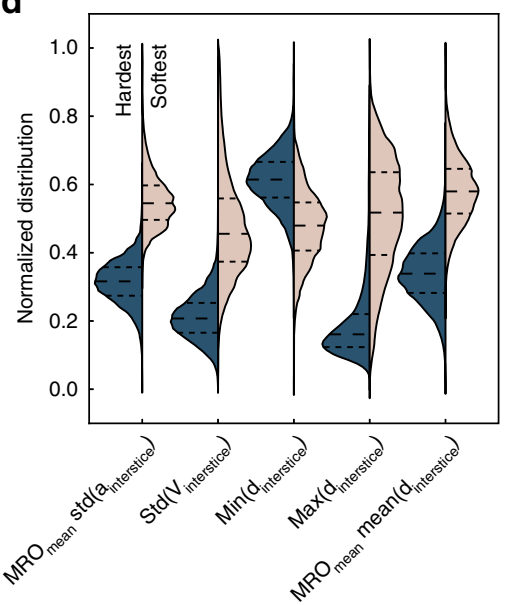

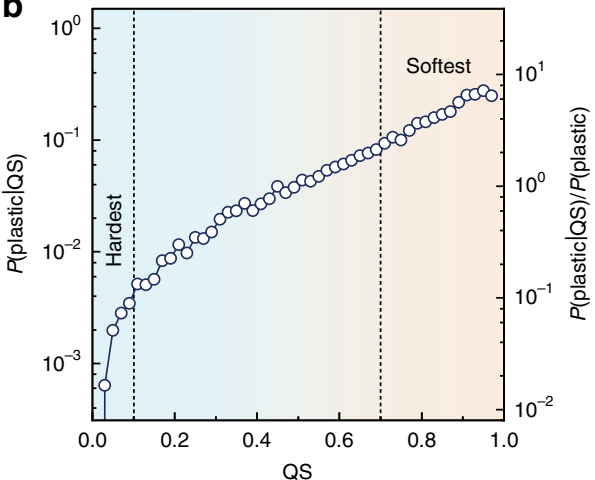

e

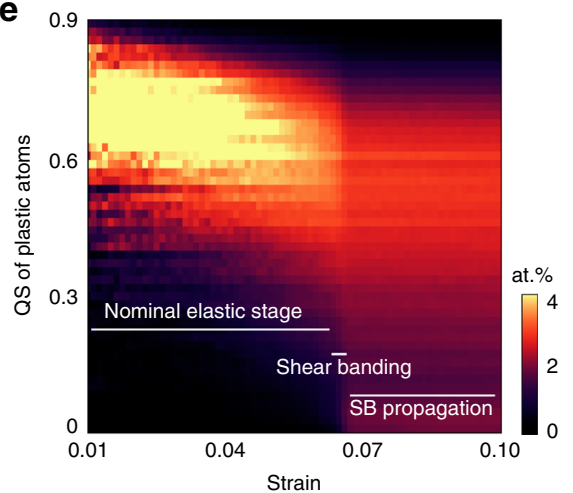

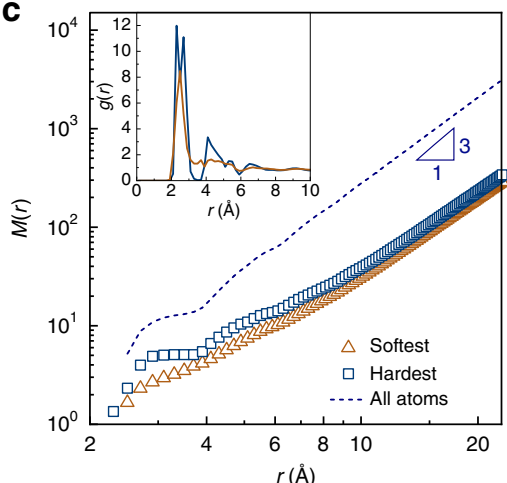

f

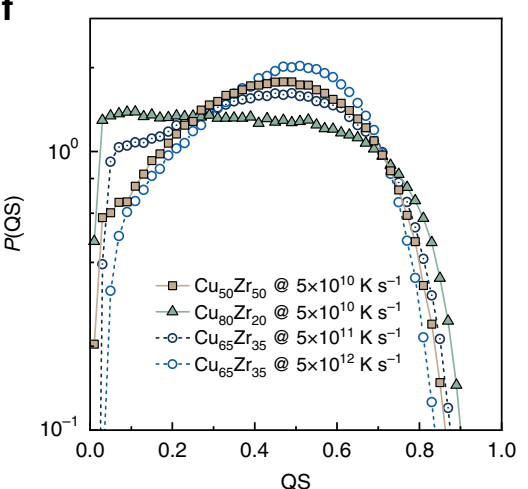

Fig. 3 Distribution of machine-learnt quench-in softness in metallic glasses. a Wide spread in the distribution of quench-in softness (QS) in $\mathrm{Cu}_{65} \mathrm{Zr}_{35}$ defines a spectrum of atomic environments within a glass. $\mathbf{b}$ The probability that an atom rearranges as a function of QS, $P$ (plastic|QS), is a strong function of QS, increasing by several orders of magnitude, from hardest atoms at low QS end to softest atoms with high QS. c Fractal dimensionality sampling in a $\log -\log$ plot for the hardest $(\mathrm{Q} S<0.1)$ and softest atoms $(\mathrm{Q} S>0.7)$ as well as all atoms, based on the power-law scaling of the mass distribution $M(r) \sim r^{D}$, where $M(r)$ denotes the number of atoms within radius $r$ centered by an atom and slope $D$ is the dimensionality. The inset shows the pair correlation function functions $g(r)$ of the hardest and softest atoms. d Violin plots of representative interstice distributions of the hardest and softest atoms, manifesting strong site environment contrasts between these two groups of characteristic atoms. e QS of the plastic atoms activated under a certain strain. The entire deformation range up to the strain of $10 \%$ with an interval of $0.125 \%$ is analyzed. The crossover between nominal elastic stage (plasticity highly dependent on high-QS atoms), shear banding and shear band propagation stages (plastic rearrangement extending across all levels of QS) is clearly shown and marked.f QS distributions of $\mathrm{Cu}_{50} \mathrm{Zr}_{50} @ 5 \times 10^{10} \mathrm{~K} \mathrm{~s}^{-1}, \mathrm{Cu}_{80} \mathrm{Zr}_{20} @ 5 \times 10^{10} \mathrm{~K} \mathrm{~s}^{-1}, \mathrm{Cu}_{65} \mathrm{Zr}_{35} @ 5 \times 10^{11} \mathrm{Ks} \mathrm{s}^{-1}$, and $\mathrm{Cu}_{65} \mathrm{Zr}_{35} @ 5 \times{ }^{1} \mathrm{O}^{12} \mathrm{~K} \mathrm{~s}^{-1}$ to reveal the effects of composition or thermal history. By varying these parameters, one can in principle tailor the site-specific plastic response of a MG. 
that the hardest atoms are more likely to form a plastic-resistant backbone that penetrate in MGs, while the soft spots are essentially localized in space with no significant correlation beyond SRO.

Next, we examine the structural traits of the hardest and softest atoms (Fig. 3d). Strong contrasts in the site environments are observed for these two groups of atoms, and a large degree of separation can be achieved even with a single interstice distribution feature. For example, the softest atoms typically have less regular, more anisotropic neighboring environments with high variance in the distance, area, and volume interstices, and this effect is more pronounced when such anisotropy is present at both short and medium ranges. As another signature, atoms with extremely low minimum bond interstice (this typically means a bond distance smaller than equilibrium distance, i.e., in the repulsive regime) and large maximum bond interstice (atoms too far apart) in the neighboring shell are more prone to be soft. In previous studies, there are two major approaches to establish the structure-plasticity relations in MGs: one focuses on the identification of locally favored atoms that are resistant to plastic deformation and behave as elastic backbone of MGs, and the other focuses on identifying flow defects or soft spots that are plastic carriers. The machine-learnt QS encompasses both ends of the spectrum and provides a complete landscape of structural deformability, from the hardest end to the softest end, in MGs.

In practice, atoms frozen in the quenched structure are gradually activated plastically with the increase of strain. We trace the QS of the activated plastic atoms as the strain is applied (Fig. 3e). We see that the progression of plastic sites indeed follows a sequence. At low strains, the plastic atoms correspond mainly to those predicted with high QS, i.e., high probability predicted by ML to be deformable. As the strain progresses, plasticity is induced at sites predicted with gradually lower QS. Thus less susceptible sites are essentially frozen until the stress is large enough to trigger the rearrangement. Throughout, a fraction of low QS atoms are also activated owing to inevitable stochastic effects and shear avalanches. ${ }^{14}$ Yet, during this entire range, the QS reasonably distinguishes plastic and non-plastic atoms. However, we also see that this sequence is abruptly disrupted by shear banding at a strain of $\sim 0.065$. Significant plastic rearrangement avalanches occur near yielding (a local rearrangement triggers others, leading to a cascade $)^{12}$, and the atoms that form shear bands cannot be identified as pre-existing structural defects. Upon shear banding, plastic rearrangement abruptly extends across all levels of QS (Fig. 3e), suggesting that a largely initial environment-independent transformation occurs along the pathway of shear bands (typical shear banding snapshots are shown in Supplementary Fig. 3). Overall, deformation occurs first in regions of high QS followed by those with low QS, and we note that QS appears to be a relevant metric only prior to the formation of shear bands.

Finally, we characterize how the QS distribution is affected by the glass composition and its thermal history (Fig. 3f). $\mathrm{Cu}_{80} \mathrm{Zr}_{20}$ has a similar QS distribution to that of $\mathrm{Cu}_{65} \mathrm{Zr}_{35}$ (Fig. 3a), while $\mathrm{Cu}_{50} \mathrm{Zr}_{50}$ is more centered around QS of 0.5 , suggesting that its site environments are less heterogeneous. The fraction of hardest atoms in $\mathrm{Cu}_{50} \mathrm{Zr}_{50}$ is also notably lower $(\sim 5.4 \%$ atoms with QS $<$ 0.1 ), suggesting a lower fraction of exceptionally plastic-resistant atoms within the glass. We then calculate the standard deviation of QS, i.e., std(QS), as an indication of structural heterogeneity, and the $\operatorname{std}(\mathrm{QS})$ of $\mathrm{Cu}_{50} \mathrm{Zr}_{50}, \mathrm{Cu}_{65} \mathrm{Zr}_{35}$, and $\mathrm{Cu}_{80} \mathrm{Zr}_{20}$ is 0.196, 0.230 , and 0.234 , respectively. This agrees with previous studies suggesting that $\mathrm{Cu}_{50} \mathrm{Zr}_{50}$ does have a lower structural and plastic heterogeneity than $\mathrm{Cu}_{65} \mathrm{Zr}_{35}{ }^{42,43}$. As to thermal history, with the increasing quenching rate, QS variation also gradually decreases (Fig. 3f), indicating a lowered structural heterogeneity developed during quenching. The fraction of hard atoms also decreases.
Overall, faster quenching results in lower structural heterogeneity that should be closer to the parent liquids.

Generalization to new compositions, quench rates, and systems. Thus far, we have trained our ML model for a specific glass system and tested it on unseen glass configurations under the same condition, i.e., same composition and quench rate. This is already a more rigorous generalization test beyond the traditional train-to-test within a single dataset. Extrapolating even further, there is a more challenging yet significant question-is it possible for the ML models to generalize across different compositions, and even different chemical systems, without re-training? This type of test has not been performed in previous glass studies ${ }^{24-26}$ and is generally challenging for all categories of ML studies ${ }^{59}$.

As a first test of generalization ability, we stayed within the same chemical system and tested all 81 possible mutual generalization pairs between the $9 \mathrm{Cu}-\mathrm{Zr}$ MGs with varying compositions and quenching rates. The tests can be generally grouped into 4 categories: (i) generalization to unseen glass configurations with same composition and quenching rate (9 tests), as a reference; (ii) generalization between glasses with the same composition but different quenching rate (18 tests); (iii) same quenching rate but different composition (18 tests); (iv) different composition (same chemical system) and quenching rate (36 tests). The generalization performance is evaluated by the difference between the AUC of a model trained specifically on a target glass with the generalized AUC achieved by applying a ML model trained for another glass to the target glass (filled violin plots in Fig. 4). Interestingly, for the case of our ML framework, we see that the generalization performances are quite close to the fitted cases for all first to fourth scenarios (the third and fourth,

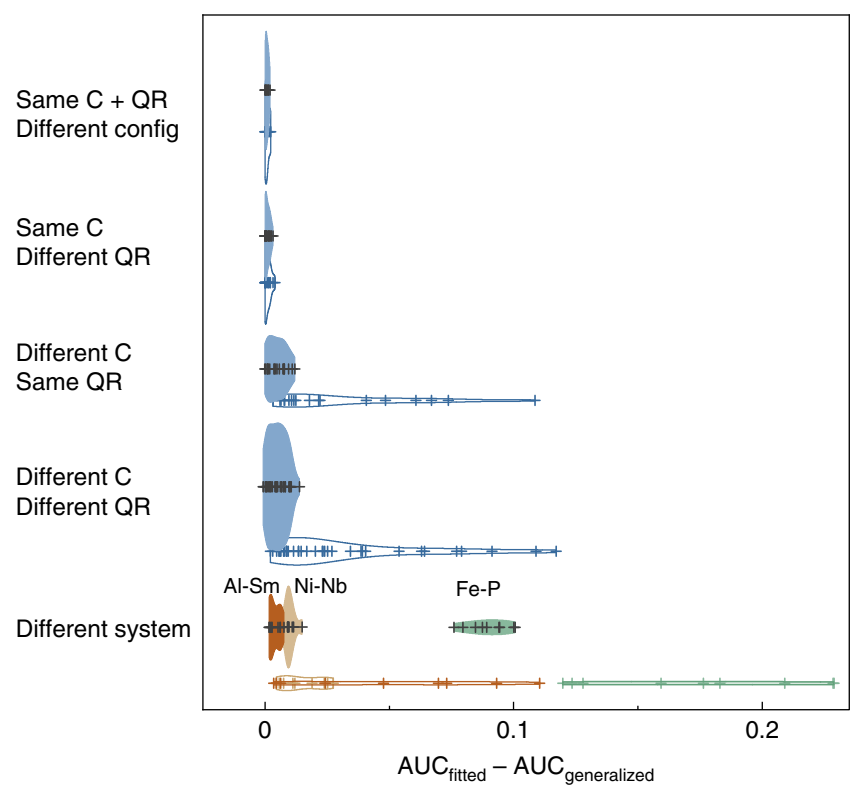

Fig. 4 Generalizability of machine learning models. Generalization performances of the ML models using our interstice distribution features (filled violin plots) and those using symmetry functions (unfilled violin plots) on five generalization scenarios: (i) same composition (abbreviated as $\mathrm{C}$ ) and same quenching rate (QR) but to new unseen configurations, (ii) same composition and different quenching rate; (iii) different composition and same quenching rate; (iv) different composition and different quenching rate; $(v)$ different system. The performance is evaluated by the difference of the generalization score and the original, same-condition score. The height of the violin plot is proportional to number of tests that fall within a performance interval and the interior shows the data points. 
i.e., transferring between different $\mathrm{Cu}-\mathrm{Zr}$ compositions, are slightly worse), with AUC decreases of $<0.015$ (see Supplementary Table 9 for typical fitted and generalization scores). This suggests a strong generalizability of our feature representation and obtained ML models between MGs within a single system.

As a more difficult problem, we further test the generalizability of our learnt models to completely different chemical systems. In addition to $\mathrm{Cu}-\mathrm{Zr}$ MGs, we extend our ML studies to $\mathrm{Ni}_{62} \mathrm{Nb}_{38}$, $\mathrm{Al}_{90} \mathrm{Sm}_{10}$, and a metal-metalloid glass $\mathrm{Fe}_{80} \mathrm{P}_{20}$ (Methods). When directly training models on these systems, we achieve AUCs of 0.737-0.775 for predicting the plastic atoms during tensile or compressive deformation (see Supplementary Table 8). The comparable accuracy suggests that our interstice representation and ML framework can apply to MGs of various structural traits. We next tested the 27 generalization pairs from the $9 \mathrm{Cu}-\mathrm{Zr} \mathrm{MGs}$ to $\mathrm{Ni}_{62} \mathrm{Nb}_{38}, \mathrm{Al}_{90} \mathrm{Sm}_{10}$, and $\mathrm{Fe}_{80} \mathrm{P}_{20}$ MGs. These tests form the fifth generalization category: (v) different chemical system (27 tests). As seen from Fig. 4, even when generalizing between different chemical systems, the models fitted in $\mathrm{Cu}-\mathrm{Zr}$ MGs can achieve good performances in the $\mathrm{Ni}-\mathrm{Nb}$ and $\mathrm{Al}-\mathrm{Sm}$ MGs with minor loss of AUCs, suggesting that the relative rankings of QS from models learnt in different MGs can be similar. The generalization to Fe-P MG is worse (with AUC decreases of $\sim 0.07-0.10$ ), which is expected as the decisive features in distinguishing the plastic and non-plastic atoms in the metal-metalloid MG are likely to be different from that of the all-metal MGs (Supplementary Table 10).

As a direct comparison, we have calculated the symmetry functions for our studied MGs based on the formulation and parameter settings of the previous works ${ }^{24-26}$ and train GBDT models on the same data and CV splits with optimized hyperparameters (see Methods section). As discussed above for the $\mathrm{Cu}-\mathrm{Zr}$ MGs, the symmetry functions overall achieved comparable AUCs (mostly with $0.01-0.02$ lower) with our features when directly training models on a specific glass (Supplementary Tables 3 and 8). Nonetheless, when transferring the
ML models, the generalizability of the two sets of models is quite different. For the ML models using symmetry functions as input, the loss in AUC on the first and second generalization tests is minor (also see Supplementary Table 9 for typical fitted and generalization scores) and comparable to our method (unfilled violin plots in Fig. 4). However, when attempting to generalize to different compositions (i.e., third and fourth tests), the AUC degrades substantially. The degradation is even worse when generalizing between different chemical systems (except for generalizing from $\mathrm{Cu}-\mathrm{Zr}$ to $\mathrm{Ni}-\mathrm{Nb}$ ). We hypothesize that this is because symmetry functions (and therefore the trained ML models) are by definition more sensitive to the length scales of the specific system. The radial part of the symmetry functions (often being more important than the angular ones) characterizes the Gaussian-smeared radial density of each species at a series of distances (Methods). Although the distances are often normalized to the equilibrium distance between one species ${ }^{24-26}$, the radial functions can still essentially be very different among systems with distinct atomic sizes and coordination environments.

Revisiting previously proposed signatures and rules. As discussed above, despite many recognized structural signatures have been proposed for $\mathrm{MGs}^{30-38}$, a standard way is still lacking to quantitatively assess and compare the predictive ability of the signatures as well as the structure-property correlation proposed $^{60}$.

In this work, we will demonstrate that ML can be used as a tool to quantitatively assess the predictive capability of candidate feature sets for MGs. By feeding each individual feature set to train a ML model, the prediction score on the same datasets and CV splits can be used as a metric of the feature set's predictive ability (Fig. 5a). As mentioned above, here we consider eight existing SRO feature sets and those sets further augmented by the MRO features generated following the coarse-graining scheme described above (Please refer to Methods for a full description of a

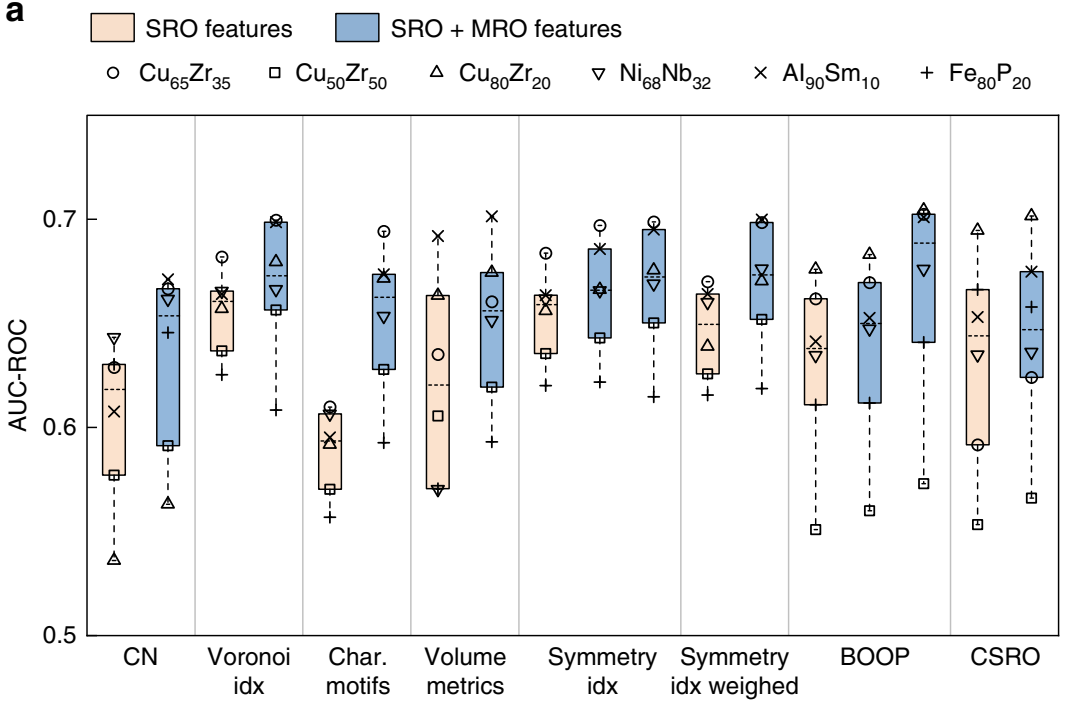

b

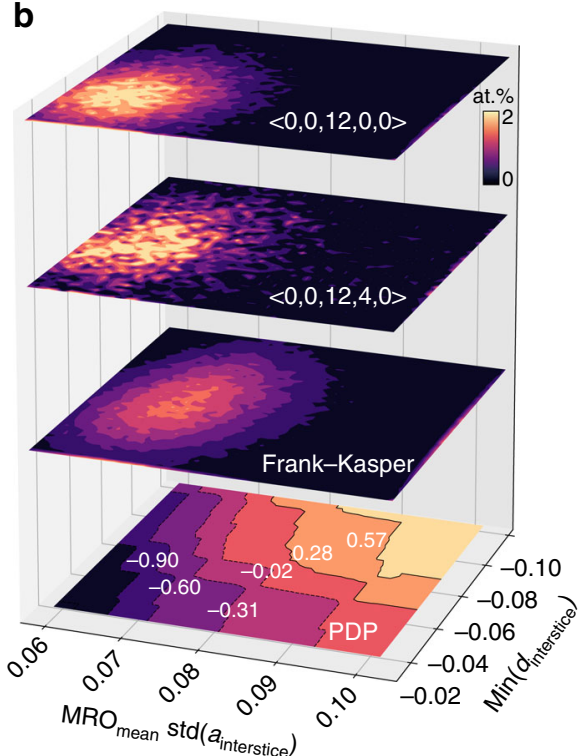

Fig. 5 Quantitatively benchmarking individual feature sets. a Quantitatively benchmarked predictive powers of individual short-range order (SRO) feature sets and the feature sets further augmented by the medium-range order (MRO) features generated following the coarse-graining technique described in this work. Please refer to Methods and Supplementary Tables 4 and 6 for a full description of the feature sets. $\mathrm{Results}$ for $\mathrm{Cu}_{65} \mathrm{Zr}_{35}, \mathrm{Cu}_{50} \mathrm{Zr}_{50}, \mathrm{Cu}_{80} \mathrm{Zr}_{20}$, $\mathrm{Ni}_{62} \mathrm{Nb}_{38}, \mathrm{Al}_{90} \mathrm{Sm}_{10}$, and $\mathrm{Fe}_{80} \mathrm{P}_{20} \mathrm{MGs}$ (quenched under $5 \times 10^{10} \mathrm{~K} \mathrm{~s}^{-1}$ ) are shown. In the box plots, bounds of the box spans from $25 \%$ to $75 \%$ percentile, dashed line represents median, and whiskers show minima and maxima of data points. b Projecting $\langle 0,0,12,0,0\rangle,\langle 0,0,12,4,0\rangle$, and Frank-Kasper clusters to the two-dimensional (2-D) partial-dependence plot (PDP) of the top two features of the ML model. Conventional descriptors only capture a small fraction of the possible input space, whereas our features and $M L$ form a more complete description of feature space. 
the feature sets). Results indicate that individual feature sets yield prediction accuracies varying notably among MGs, even among different compositions within a single $\mathrm{Cu}-\mathrm{Zr}$ system (Fig. 5a). Overall, the plastic atoms in $\mathrm{Cu}_{50} \mathrm{Zr}_{50}$ and $\mathrm{Fe}_{80} \mathrm{P}_{20}$ are the most difficult to predict for these feature sets. Augmenting the SRO features by the coarse-grained MRO features overall improves the predictions.

Interestingly, we find that the SRO characteristic motif features that describe whether an atom's neighbors form icosahedra $<0,0,12,0,0\rangle$ (or $<0,0,12,0\rangle$ if omitting facets with 7 edges and more), $\langle 0,0,12,4,0\rangle$, or Frank-Kasper clusters ${ }^{31}$ generally have the lowest AUCs among all SRO feature sets. Despite these clusters have been identified to be of the most stable clusters in MGs, especially the $\mathrm{Cu}-\mathrm{Zr}$ MGs, not forming any of these clusters does not mean that the atom is not stable. Meanwhile, as clusters with the same Voronoi indices could have different packing, falling in one of these clusters does not guarantee a low plastic susceptibility. Both factors restrict the plastic/non-plastic distinguishability of these motifs. However, when extended to MRO, the predictive power of the characteristic motif features has greatly enhanced, e.g., AUC increasing from 0.610 (SRO) to 0.694 (SRO + MRO) for $\mathrm{Cu}_{65} \mathrm{Zr}_{35}$. This evidences that the cluster-cluster connection of these motifs in the medium range could be more important, as discussed in previous studies ${ }^{42,43}$.

We further plot the two-dimensional partial dependence plot $(\mathrm{PDP})^{56}$ of our top two features and overlay the positions in that feature space for atom sites corresponding to $\langle 0,0,12,0,0\rangle$ icosahedra, $\langle 0,0,12,4,0\rangle$, and Frank-Kasper clusters (Fig. 5b). Owing to the difficulty of visualizing the decision boundary in high-dimensional feature space, PDP offers a mechanism to resolve the effect of specific features by marginalizing the model output over other features, in essence measuring how the model prediction changes (on average) as a function of the target features ${ }^{56}$. We see that these characteristic motifs are indeed residing in the regions that correspond to low plastic susceptibility (negative partial dependence). This confirms the increased stability of these long-proposed motifs. Nonetheless, all the motifs are distributed only in a narrow portion of the entire feature space, while in contrast, our features form a more complete description of feature space (Fig. 5b). These interstice features (together with the other 13 features not shown) capture not only the stable nature of regions of feature space corresponding to these characteristic motifs but also the effect of less conventional feature space (e.g., unstable SRO but stable MRO may also stabilize the atoms) to form the final decision boundary. We refer to Supplementary Figs. 8-25 for more complete analyses of PDPs to interpret ML models obtained with each SRO or MRO feature set. Furthermore, we reiterate that further augmenting our interstice representation with these SRO or generated MRO feature sets leads to a negligible AUC increase (Supplementary Tables 5 and 7).

\section{Discussion}

The present work can also have impact on the design of MGs with tailored mechanical behaviors. Our findings demonstrate that much of plasticity within the elastic regime (prior to shear band formation) is controlled by QS that is determined by the initial glass configuration, rather than by complex dynamics. Thus, any processing route that can modify the glass structure could potentially tailor the distribution of QS in the materials (e.g., as in Fig. 3f) and tailor the resultant deformation responses. For instance, it has been proposed that, to make MGs ductile, a larger density of plastic units is preferred, and plausible routes can be changing the thermal history, such as ultrafast quenching, rejuvenating glass structure through thermomechanical cycling, or applying irradiation techniques to transform the glass into more deformable structural state ${ }^{61}$. One can use the ML models to get some quick estimates on the plastic heterogeneity of candidate configurations derived by processing routes of interest.

Furthermore, as our ML models can generalize well to unseen glass configurations, even of different chemical systems, they can be applied even in the absence of simulated configurations tailored to the specific system of interest and be applied directly to glass configurations inversely generated from experimental diffraction or extended x-ray absorption fine structure spectra using techniques such as reverse Monte Carlo ${ }^{34}$. We can also use the ML models to perform tests on virtual geometries. For example, one could easily test whether certain perturbations or transformations to an initial geometry greatly change the plastic susceptibility or not, without performing any explicit simulations. This could help locate some optimal structures that minimize or maximize some chosen metric, such as the degree of heterogeneity in plastic susceptibility. Herein, the use of ML could help accelerate the development cycle of glasses with targeted mechanical behaviors. We also note that the ML-learnt mapping between the interstice distribution and plastic heterogeneity are much more generalizable between MGs that only contain metallic elements than to metallic-metalloid MGs (Fig. 4). This can be attributed to their different atomic interactions: one principally includes metallic bonds and another involve more directional covalent bond contributions. Thus generalization performance should be expected to vary depending on the type of system under investigation.

In this work, we use data from a single compressive/tensile deformation simulation to fit the ML models. We note that using athermal quasi-static deformation ${ }^{62}$ or iso-configurational ensemble technique ${ }^{21}$ to construct the datasets may reduce thermal fluctuations and stochastic effects and improve the prediction scores. We choose to use the current setting to mimic the real experimental deformation in which thermal fluctuations and stochastic effects do play a role. Here we show that ML is indeed capable of learning a mapping that best explains the data (even if there are some noises inside), and the learnt model can even be generalized to completely unseen compositions or chemical systems with appropriate representation and learning protocols.

To summarize, the heterogeneity of atomic environments in MGs makes it formidably challenging to predict their response to external stimuli at the atomic scale. In this work, we demonstrate that focusing on the short- and medium-range distribution of interstitial spaces (distances, areas, and volumes) and applying ML can help form an interpretable and generalizable model to predict the atomic-scale response to mechanical stress for several different systems. In addition to deformation, the ML framework we describe is readily generalizable to the studies of other sitedependent properties and could also be applied to other important physical processes, such as thermal activation, glass transition, and relaxation.

\section{Methods}

Featurizing interstice distribution in MGs. We use two methods for determining near neighbors: Voronoi tessellation and cutoff distances. The convex hull is derived using scipy ${ }^{40}$ (based on qhull library ${ }^{41}$ ) with qhull option of "Qt" (triangulated output), and all facets will be simplicial.

The procedure of calculating the distance interstice between center atom $\mathrm{O}$ and neighbor $\mathrm{A}$ is as follows: (i) calculate the distance $d_{\text {bond }}$ between $\mathrm{O}$ and $\mathrm{A}$; (ii) calculate the atom-packed distance $d_{\text {pack }}$ as the sum of atom sizes as $\sum_{\mathrm{O}, \mathrm{A}} R_{i}$, where $R_{i}$ is radius of atom at site $i$; (iii) derive the distance interstice as ( $d_{\mathrm{bond}}-$ $\left.d_{\text {pack }}\right) / d_{\text {bond. }}$.

The procedure of deriving the area interstice of facet $\mathrm{ABC}$ on the convex hull: (i) calculate the triangle area $a_{\text {triangle; }}$ (ii) calculate the angle $\theta_{i}$ of each vertex $i$ as 
$\arccos \left(\frac{\mathbf{r}_{i j} \cdot \mathbf{r}_{i k}}{\left|\mathbf{r}_{i j}\right| \mathbf{r}_{i k} \mid}\right)$; (iii) calculate the atom-packed circular sector area $a_{\text {pack }}$ as

$\sum_{\mathrm{A}, \mathrm{B}, \mathrm{C}} R_{i}^{2} \theta_{i} / 2$; (iv) derive the area interstice as $\left(a_{\text {triangle }}-a_{\text {pack }}\right) / a_{\text {triangle }}$.

The procedure of computing the volume interstice of tetrahedron formed by center atom $\mathrm{O}$ with facet $\mathrm{ABC}$ : (i) calculate the tetrahedron volume $v_{\text {tetrahedron; }}$ (ii) calculate the solid angle $\Omega_{i}$ of each vertex $i$ as

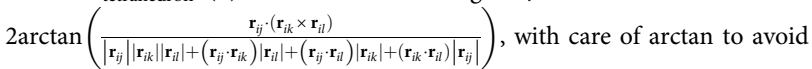
negative value; (iii) calculate the atom-packed cone volume $v_{\text {pack }}$ as $\sum_{\text {O.A,B,C }} R_{i}^{3} \Omega_{i} / 3$; (iv) derive the volume interstice as $\left(v_{\text {tetrahedron }}-v_{\text {pack }}\right) / v_{\text {tetrahedron }}$

In this work, we use atomic radii from Miracle et $\mathrm{al}^{35}$. One can also use the equilibrium distance estimates from pair correlation functions or other sources. The values of interstices would be affected by the atomic radii, but this will not affect the performance of $\mathrm{ML}$ as long as the classes are distinguishable.

In essence, this representation is also applicable to crystalline interstices. As an example, for a one-component bcc structure, supposing that each atom perfectly touch the 8 nearest neighbors (neglecting the six second-nearest neighbors), the distance, area, and volume interstice vector would be $[0, \ldots, 0]_{\text {length }=8},[0.41, \ldots$, $0.41]_{\text {length }=12}$, and $[0.32, \ldots, 0.32]_{\text {length }=12}$. It follows that the mean, min, and $\max$ of the distance, area, and volume interstice distribution features will be $0,0.41$, and 0.32 (it is known that the volumetric packing factor of a perfect bcc structure is 0.68 ), respectively, and the standard deviations will all be 0 .

Machine learning. We use GBDT as our ML algorithm and the hyperparameters searched in this work can be found in Supplementary Table 1. The GBDT model is trained on the plastic heterogeneity data at a strain of $4.0 \%$ to learn to classify the atomic environments back in the undeformed configuration as plastic or nonplastic. For $\mathrm{Cu}-\mathrm{Zr} \mathrm{MGs}$, a single model is fitted for both $\mathrm{Cu}$ and $\mathrm{Zr}$ atoms (two types of atoms in one model), and for $\mathrm{Ni}_{62} \mathrm{Nb}_{38}, \mathrm{Al}_{90} \mathrm{Sm}_{10}$, and $\mathrm{Fe}_{80} \mathrm{P}_{20}$, the model is for the host (majority) atoms only. Owing to the localized plasticity of glasses under low temperatures or slow strain rates, the non-plastic atoms heavily outnumber the plastic atoms (approximately 3.5-6.0\% of atoms are plastic at a strain of $4.0 \%$ ). We deal with the between-class imbalance by random equal undersampling to create a balanced dataset. After performing fivefold CV on the sampled datasets, we generalize the obtained models to the unseen glass configuration and calculate the average scoring metric and average probability estimates as QS. In this work, we use AUC-ROC on the unseen glass configuration as the scoring metric, instead of the recall used in previous studies (although we also report recall for comparison purposes when needed)

We propose to report AUC along with the recall used in previous works because (i) AUC evaluates the tradeoff between true positive rate against false positive rate as a function of chosen threshold, and thus balances the tradeoff between over- and under-predicting the plastic atoms, and does not depend on optimizing a specific prediction threshold (as with precision, recall, or f1 score); (ii) the AUC score can be interpreted as the probability that a true positive atom (plastic) is assigned a higher plastic probability (ranks higher than) a true negative atom (non-plastic). ${ }^{63}$ This is in accord with the scenario suggested by glass dynamics, which indicates that many structurally soft atoms in the glasses may not be activated under each deformation test, and thus a good model would aim to increase the possibility that the activated plastic atoms are ranked higher than non-plastic ones; (iii) AUC-ROC is robust with the imbalanced data ${ }^{64}$ (see Supplementary Fig. 6 for an illustration). We note that we prefer AUC-ROC over area under the precision-recall curve because AUC-ROC gives equal weight to plastic and non-plastic atom classification.

Symmetry functions. Symmetry functions are first proposed to fit ML interatomic potential ${ }^{28,29}$ and later employed to represent the atomic environment in disordered materials ${ }^{24-26}$. For an atom $i$ in a binary A-B system, the radial and angular symmetry functions are described as ${ }^{24-26}$,

$$
\begin{gathered}
G_{X}(i ; r)=\sum_{j \in X} e^{-\left(r_{i j}-r\right)^{2} / 2 \sigma^{2}} \\
\psi_{X Y}(i ; \xi, \lambda, \zeta)=\sum_{j \in X} \sum_{k \in Y} e^{-\left(r_{i j}^{2}+r_{i k}^{2}+r_{j k}^{2}\right) / \xi^{2}}\left(1+\lambda \cos \theta_{i j k}\right)^{\zeta}
\end{gathered}
$$

here $X$ and $Y$ denote the atom species in the system: in Eq. 2, $X$ can be A or B, and in Eq. 3, $(X, Y)$ can be $(\mathrm{A}, \mathrm{A}),(\mathrm{A}, \mathrm{B})$, or $(\mathrm{B}, \mathrm{B}) . r_{i j}$ is the distance between atoms $i$ and $j, \theta_{i j k}$ is the angle between $\mathbf{r}_{i j}$ and $\mathbf{r}_{i k}, \sigma$ is a constant that is often set as the bin size of $r$, and $r, \xi, \lambda$, and $\xi$ are variable constants. The sums are taken over atom pairs whose distance is within a cutoff $R^{c}$.

The radial part characterizes the Gaussian-smeared radial density of species $X$ at each $r$ (proportional to $r^{2} g(r)$ if $\sigma \rightarrow 0$, where $g(r)$ is the radial density function around atom $i$ ), and the angular part characterizes bond orientations following the formulation of Behler et al. ${ }^{28}$. Varying $r, \xi, \lambda$, and $\xi$ generates a group of symmetry functions that characterize the site environments around each atom ${ }^{24-26}$. In this work, we follow the settings of $r, \xi, \lambda$, and $\zeta$ in previous works ${ }^{24-26}$ to derive the symmetry. For example, for an atom $i$ in $\mathrm{Cu}-\mathrm{Zr} \mathrm{MGs}$, we derive 100 radial functions (50 for $i-\mathrm{Cu}$ and 50 for $i-\mathrm{Zr}$ ) by varying $r$ from 0 to $5.0 \times \mathrm{Cu}-\mathrm{Cu}$ equilibrium distance (sum of metallic radii) with increments of $0.1 \times \mathrm{Cu}-\mathrm{Cu}$ equilibrium distance, and 66 angular functions ( 22 for $\mathrm{Cu}-i-\mathrm{Cu}, \mathrm{Cu}-i-\mathrm{Zr}$, and $\mathrm{Zr}-i-\mathrm{Zr}$, respectively) by using 22 sets of $\xi, \lambda$, and $\xi$ for each atom. Please refer to the original papers ${ }^{24-26}$ for details of parameter settings. We then take the 166 features as input and train GBDT models on the same datasets and CV splits with our interstice features.

Benchmarked structural signatures and their MRO version. To conduct an extensive benchmarking of candidate feature sets in the field of MGs (Fig. 5a), we have featurized eight SRO feature sets and further extract their MRO features following the coarse-graining technique described above (Eq. 1) and combine them with the SRO features to explore their predictive capability if being extended from SRO to MRO. The SRO feature sets as well as the statistical types used in generating the MRO ones are listed as follows. Some feature sets such as $i$-fold symmetry and BOOP have two ways to extend to MRO, and both are included in the benchmarks. The order is the same with that (from left to right) in Fig. 5a, with the number of features in parentheses.

i. $\mathrm{CN}_{\text {Voro/Dist }}(2)$ : Coordination number by Voronoi tessellation ${ }^{30}$ or by cutoff distance;

MRO $\mathrm{CN}_{\text {Voro/Dist }}(8)$ : mean, std, min, and max.

ii. Voronoi $\operatorname{idx}_{3 \ldots 7}(5):\left\{n_{i}\right\}$ where $n_{i}$ is the number of $i$-edged facets $(i$ in the range of 3-7) in the Voronoi polyhedra ${ }^{30}$. MRO Voronoi $\operatorname{idx}_{3 \ldots 7}(20)$ : mean, std, min, and max.

iii. Characteristic motifs (4): One-hot encoded signatures of whether a cluster belongs to $\langle 0,0,12,0,0\rangle,\langle 0,0,12,4,0\rangle,\langle 0,0,12,0,0\rangle \|\langle 0,0,12,4,0\rangle$, or Frank-Kasper-type clusters ${ }^{31}$;

MRO Characteristic motifs (12): sum, mean, and std (min and max are not helpful, as they are one-hot encoded features and the min and max over the neighbors would be 0 and 1 in almost all cases).

iv. Volume metrics (3): Cluster packing efficiency ${ }^{34}$, atomic packing efficiency ${ }^{35}$, and the ratio of the atomic volume to the Voronoi polyhedron volume around each site;

MRO Volume metrics (12): mean, std, min, and max.

v. $i$-fold symm $\operatorname{idx}_{3 \ldots 7}(5): n_{i} / \sum_{i=3}^{7} n_{i}$ where $n_{i}$ is Voronoi index ( $i$ in the range of 3-7), reflecting the strength of $i$-fold symmetry in local sites ${ }^{36}$;

MRO Avg. $i$-fold symm idx $\mathrm{id}_{3 . .7}$ (5): $\sum_{m=0}^{\mathrm{NN}} n_{i}^{m} / \sum_{m=0}^{\mathrm{NN}} \sum_{i=3}^{7} n_{i}^{m}$, where $n_{i}$ denotes the number of $i$-edged facets of the Voronoi polyhedra and $m$ iterates over each neighbor;

MRO $i$-fold symm $\operatorname{idx}_{3 \ldots 7}(20)$ : mean, std, min, and max.

vi. weighted $i$-fold symm idx $\mathrm{x}_{3 . .7}$ (5): using Voronoi facet areas as weights in calculating the $i$-fold symm idx in $_{3 . .7}$; MRO Weighted $i$-fold symm idx $x_{3} \ldots 7$ (20): mean, std, min, and max.

vii. BOOP $\mathrm{q}_{4} \ldots 10$-Voro/Dist and $\mathrm{w}_{4} \ldots 10$-Voro/Dist $(16)$ : Lowest- and higher-order rotation-invariant $q_{l}$ and $w_{1}(l=4,6,8$, and 10$)$ of the $l$ th moment in a multipole expansion of the bond vector distribution on a unit sphere ${ }^{33}$; Coarse-grained BOOP (16): Coarse-grained ${ }^{65}$ lowest-order and higherorder rotation-invariant $\overline{q_{l}}$ and $\overline{w_{l}}(l=4,6,8$, and 10$)$;

MRO BOOP $\mathrm{q}_{4 \ldots 10-V o r o / D i s t}$ and $\mathrm{w}_{4 \ldots 10-V_{0} / \text { Dist }}$ (64): mean, std, min, and max.

viii. $\mathrm{CSRO}_{\text {Voro/Dist }}(9)$ : Element type, the number, and the deviation of local chemistry with nominal composition (Warren-Cowley parameters ${ }^{37,38}$ ); $\mathrm{CMRO}_{\text {Voro/Dist }}(32)$ : mean, std, min, and max.

These SRO features are among the most recognized signatures in the field of MGs. Voronoi tessellation (signified by subscript "Voro") and cutoff distance (subscript "Dist") are both used to define neighbors in calculating $\mathrm{CN}$, BOOP, and CSRO. One can also refer to Supplementary Tables 4 and 6 for a more detailed description of the features. These SRO features can be calculated from amlearn and the MRO features can be derived using helper statistical functions in amlearn or matminer.

Liquid quenching and deformation simulation. We simulate liquid melt quenching and deformation of $\mathrm{Cu}_{x} \mathrm{Zr}_{1-x}\left(x=50,65\right.$, and 80 at.\%), $\mathrm{Ni}_{62} \mathrm{Nb}_{38}$ $\mathrm{Al}_{90} \mathrm{Sm}_{10}$, and $\mathrm{Fe}_{80} \mathrm{P}_{20} \mathrm{MGs}$ using molecular dynamics simulations. We use three quenching rates of $5 \times 10^{10}, 5 \times 10^{11}$, and $5 \times 10^{12} \mathrm{~K} \mathrm{~s}^{-1}$ for Cu-Zr MGs and $5 \times 10^{10} \mathrm{~K} \mathrm{~s}^{-1}$ for all other MGs. We construct three large slab samples for each $\mathrm{Cu}-\mathrm{Zr}$ MG, each of which contains 345,600 atoms with dimensions $\sim 120(X) \times 24$ $(Y) \times 240(Z) \AA^{3}$. Data from two glass samples are concatenated, equally undersampled, and used in fivefold CV training the ML models, whereas the remaining sample is set aside for rigorous generalization tests. For $\mathrm{Ni}_{62} \mathrm{Nb}_{38}, \mathrm{Al}_{90} \mathrm{Sm}_{10}$, and $\mathrm{Fe}_{80} \mathrm{P}_{20}$ MGs, we construct samples of 131,072 atoms. We use LAMMPS ${ }^{66}$ and EAM potentials as in refs. ${ }^{51,53-55}$. The timestep is $1 \mathrm{fs}$. During simulation, the initial configuration is built by randomly substituting into an fcc $\left(\mathrm{Cu}-\mathrm{Zr}, \mathrm{Ni}_{62} \mathrm{Nb}_{38}\right.$, and $\left.\mathrm{Al}_{90} \mathrm{Sm}_{10}\right)$ or bcc $\left(\mathrm{Fe}_{80} \mathrm{P}_{20}\right)$ lattice. The samples are annealed at $2000 \mathrm{~K}$ for $1 \mathrm{~ns}$, quenched to $50 \mathrm{~K}$ with each quenching rate, and relaxed at $50 \mathrm{~K}$ for $1 \mathrm{~ns}$.

After quenching, the $\mathrm{Cu}-\mathrm{Zr}$ MGs are compressed along $Z$ axis under a strain rate of $2.5 \times 10^{7} \mathrm{~s}^{-1}$ in a quasi-static mode (constantly apply a small strain and then relax, up to the strain of $10 \%$ ) at a low temperature of $50 \mathrm{~K}$ (see Supplementary Fig. 3 for typical stress-strain curves). Periodic boundary conditions (PBCs) are imposed in $Y$ and $Z$ axes and free surfaces are applied along $X$ axis to allow shear offsets. For $\mathrm{Ni}_{62} \mathrm{Nb}_{38}, \mathrm{Al}_{90} \mathrm{Sm}_{10}$, and $\mathrm{Fe}_{80} \mathrm{P}_{20}$, we simulate both tensile and compressive deformation with strain rates of $2.5 \times 10^{7} \mathrm{~s}^{-1}$ and $1.0 \times 10^{8} \mathrm{~s}^{-1}$ as well as with PBCs in all directions. After feature extraction, we 
select atoms of $\sim 10-20 \AA$ away from the surfaces or deformation ends to construct the ML datasets.

We also note that many glass deformation papers ${ }^{42,43}$ employ a strategy of quenching a small cell and replicating it to build a large simulation cell for deformation simulation. This strategy can save a large amount of time and seems to have no significant effects on deformation behaviors. However, in generating data for ML, this will generate replicated site environments that create the potential for ML information leakage, overfitting, and overestimation of score. In this work, we simulate large, un-replicated samples to guarantee the non-duplication of site environments for ML.

\section{Data availability}

The datasets used in this work are available in figshare with the DOI of https://doi.org/ 10.6084/m9.figshare.7941014.v2.

\section{Code availability}

The featurization and ML codes can be publicly found in our open-source packages amlearn (https://github.com/Qi-max/amlearn) and matminer (https://github.com/ hackingmaterials/matminer).

Received: 7 April 2019; Accepted: 4 November 2019;

Published online: 05 December 2019

\section{References}

1. Johnson, W. L. Bulk glass-forming metallic alloys: science and technology. MRS Bull. 24, 42-56 (1999).

2. Inoue, A. Stabilization of metallic supercooled liquid and bulk amorphous alloys. Acta Mater. 48, 279-306 (2000).

3. Lubchenko, V. \& Wolynes, P. G. Theory of structural glasses and supercooled liquids. Annu. Rev. Phys. Chem. 58, 235-266 (2006).

4. Alexander, S. Amorphous solids: their structure, lattice dynamics and elasticity. Phys. Rep. 296, 65-236 (1998).

5. Dyre, J. C. Colloquium: The glass transition and elastic models of glassforming liquids. Rev. Mod. Phys. 78, 953-972 (2006).

6. Cheng, Y. Q. \& Ma, E. Atomic-level structure and structure-property relationship in metallic glasses. Prog. Mater. Sci. 56, 379-473 (2011).

7. Berthier, L. \& Biroli, G. Theoretical perspective on the glass transition and amorphous materials. Rev. Mod. Phys. 83, 587-645 (2011).

8. Wang, W. H., Dong, C. \& Shek, C. H. Bulk metallic glasses. Mater. Sci. Eng. $R$ Rep. 44, 45-90 (2004).

9. Argon, A. S. Plastic deformation in metallic glasses. Acta Metall. 27, 47-58 (1979).

10. Falk, M. L. \& Langer, J. S. Dynamics of viscoplastic deformation in amorphous solids. Phys. Rev. E 57, 7192-7205 (1998).

11. Trexler, M. M. \& Thadhani, N. N. Mechanical properties of bulk metallic glasses. Prog. Mater. Sci. 55, 759-839 (2010).

12. Greer, A. L., Cheng, Y. Q. \& Ma, E. Shear bands in metallic glasses. Mater. Sci. Eng. R Rep. 74, 71-132 (2013).

13. Liu, Y. H. et al. Super plastic bulk metallic glasses at room temperature. Science 315, 1385-1388 (2007).

14. Hufnagel, T. C., Schuh, C. A. \& Falk, M. L. Deformation of metallic glasses: recent developments in theory, simulations, and experiments. Acta Mater. 109, 375-393 (2016).

15. Wisitsorasak, A. \& Wolynes, P. G. Dynamical theory of shear bands in structural glasses. Proc. Natl Acad. Sci. 114, 1287-1292 (2017).

16. Widmer-Cooper, A., Perry, H., Harrowell, P. \& Reichman, D. R. Irreversible reorganization in a supercooled liquid originates from localized soft modes. Nat. Phys. 4, 711-715 (2008).

17. Tanguy, A., Mantisi, B. \& Tsamados, M. Vibrational modes as a predictor for plasticity in a model glass. EPL 90, 16004 (2010).

18. Manning, M. L. \& Liu, A. J. Vibrational modes identify soft spots in a sheared disordered packing. Phys. Rev. Lett. 107, 108302 (2011).

19. Patinet, S., Vandembroucq, D. \& Falk, M. L. Connecting local yield stresses with plastic activity in amorphous solids. Phys. Rev. Lett. 117, 045501 (2016).

20. Zylberg, J., Lerner, E., Bar-Sinai, Y. \& Bouchbinder, E. Local thermal energy as a structural indicator in glasses. Proc. Natl Acad. Sci. 114, 7289-7294 (2017).

21. Widmer-Cooper, A., Harrowell, P. \& Fynewever, H. How reproducible are dynamic heterogeneities in a supercooled liquid? Phys. Rev. Lett. 93, 135701 (2004).

22. Larini, L., Ottochian, A., De Michele, C. \& Leporini, D. Universal scaling between structural relaxation and vibrational dynamics in glass-forming liquids and polymers. Nat. Phys. 4, 42 (2007).

23. Ding, J. et al. Universal structural parameter to quantitatively predict metallic glass properties. Nat. Commun. 7, 13733 (2016).
24. Cubuk, E. D. et al. Identifying structural flow defects in disordered solids using machine-learning methods. Phys. Rev. Lett. 114, 108001 (2015).

25. Cubuk, E. D. et al. Structure-property relationships from universal signatures of plasticity in disordered solids. Science 358, 1033-1037 (2017).

26. Schoenholz, S. S., Cubuk, E. D., Sussman, D. M., Kaxiras, E. \& Liu, A. J. A structural approach to relaxation in glassy liquids. Nat. Phys. 12, 469-471 (2016).

27. Ma, X. et al. Heterogeneous activation, local structure, and softness in supercooled colloidal liquids. Phys. Rev. Lett. 122, 28001 (2019).

28. Behler, J. \& Parrinello, M. Generalized neural-network representation of highdimensional potential-energy surfaces. Phys. Rev. Lett. 98, 146401 (2007).

29. Bartók, A. P., Kondor, R. \& Csányi, G. On representing chemical environments. Phys. Rev. B 87, 184115 (2013).

30. Okabe, A., Boots, B., Sugihara, K. \& Chiu, S. N. Spatial Tesselations. Concepts and Applications of Voronoi Diagrams (John Wiley \& Sons, 2009).

31. Frank, F. C. \& Kasper, J. S. Complex alloy structures regarded as sphere packings. I. Definitions and basic principles. Acta Crystallogr. 11, 184-190 (1958).

32. Sheng, H. W., Luo, W. K., Alamgir, F. M., Bai, J. M. \& Ma, E. Atomic packing and short-to-medium-range order in metallic glasses. Nature 439, 419-425 (2006).

33. Steinhardt, P. J., Nelson, D. R. \& Ronchetti, M. Bond-orientational order in liquids and glasses. Phys. Rev. B 28, 784-805 (1983).

34. Yang, L. et al. Atomic-scale mechanisms of the glass-forming ability in metallic glasses. Phys. Rev. Lett. 109, 105502 (2012).

35. Laws, K. J., Miracle, D. B. \& Ferry, M. A predictive structural model for bulk metallic glasses. Nat. Commun. 6, 8123 (2015).

36. Peng, H. L., Li, M. Z. \& Wang, W. H. Structural signature of plastic deformation in metallic glasses. Phys. Rev. Lett. 106, 135503 (2011)

37. Cowley, J. M. An approximate theory of order in alloys. Phys. Rev. 77, 669-675 (1950).

38. Warren, B. E. X-ray diffraction. Analysis 1, 402 (1990).

39. Tilley, R. J. D. Defects in Solids, Vol. 4 (John Wiley \& Sons, 2008).

40. Jones, E., Oliphant, T. \& Peterson, P. SciPy: open source scientific tools for Python (2001).

41. Barber, C. B., Dobkin, D. P., Dobkin, D. P. \& Huhdanpaa, H. The quickhull algorithm for convex hulls. ACM Trans. Math. Softw. 22, 469-483 (1996).

42. Lee, M., Lee, C. M., Lee, K. R., Ma, E. \& Lee, J. C. Networked interpenetrating connections of icosahedra: effects on shear transformations in metallic glass. Acta Mater. 59, 159-170 (2011).

43. Li, M., Wang, C. Z., Hao, S. G., Kramer, M. J. \& Ho, K. M. Structural heterogeneity and medium-range order in Zrx Cu100-x metallic glasses. Phys. Rev. B 80, 184201 (2009).

44. Soklaski, R., Nussinov, Z., Markow, Z., Kelton, K. F. \& Yang, L. Connectivity of icosahedral network and a dramatically growing static length scale in $\mathrm{Cu}-\mathrm{Zr}$ binary metallic glasses. Phys. Rev. B 87, 184203 (2013).

45. Krizhevsky, A., Sutskever, I. \& Hinton, G. E. ImageNet classification with deep convolutional neural networks. Adv. Neural Inf. Process. Syst. 25, 1097-1105 (2012).

46. Ward, L. et al. Matminer: An open source toolkit for materials data mining. Comput. Mater. Sci. 152, 60-69 (2018).

47. Peterson, P. F2PY: a tool for connecting Fortran and Python programs. Int. J. Comput. Sci. Eng. 4, 296-305 (2009).

48. Tang, M. B., Zhao, D. Q., Pan, M. X. \& Wang, W. H. Binary Cu-Zr bulk metallic glasses. Chin. Phys. Lett. 21, 901-903 (2004).

49. Ding, J., Patinet, S., Falk, M. L., Cheng, Y. \& Ma, E. Soft spots and their structural signature in a metallic glass. Proc. Natl Acad. Sci. 111, 14052-14056 (2014).

50. Xu, D., Lohwongwatana, B., Duan, G., Johnson, W. L. \& Garland, C. Bulk metallic glass formation in binary Cu-rich alloy series - $\mathrm{Cu} 100-\mathrm{xZrx}(\mathrm{x}=34$, $36,38.2,40$ at.\%) and mechanical properties of bulk Cu64Zr36 glass. Acta Mater. 52, 2621-2624 (2004).

51. Mendelev, M. I., Rehbein, D. K., Ott, R. T., Kramer, M. J. \& Sordelet, D. J. Computer simulation and experimental study of elastic properties of amorphous Cu-Zr alloys. J. Appl. Phys. 102, 093518 (2007).

52. Xia, L., Li, W. H., Fang, S. S., Wei, B. C. \& Dong, Y. D. Binary Ni-Nb bulk metallic glasses. J. Appl. Phys. 99, 26103 (2006)

53. Zhang, Y., Ashcraft, R., Mendelev, M. I., Wang, C. Z. \& Kelton, K. F. Experimental and molecular dynamics simulation study of structure of liquid and amorphous Ni62Nb38 alloy. J. Chem. Phys. 145, 204505 (2016).

54. Mendelev, M. I. et al. Development of interatomic potentials appropriate for simulation of devitrification of Al90Sm10 alloy. Model. Simul. Mater. Sci. Eng. 23, 45013 (2015).

55. Ackland, G. J., Mendelev, M. I., Srolovitz, D. J., Han, S. \& Barashev, A. V. Development of an interatomic potential for phosphorus impurities in iron. J. Phys. Condens. Matter 16, S2629-S2642 (2004).

56. Friedman, J. H. Greedy function approximation: a gradient boosting machine. Ann. Stat. 29, 1189-1232 (2001).

57. Falconer, K. Fractal Geometry: Mathematical Foundations and Applications (John Wiley \& Sons, 2004). 
58. Ding, J., Asta, M. \& Ritchie, R. O. On the question of fractal packing structure in metallic glasses. Proc. Natl Acad. Sci. 114, 8458-8463 (2017).

59. Ward, L., Agrawal, A., Choudhary, A. \& Wolverton, C. A general-purpose machine learning framework for predicting properties of inorganic materials. npj Comput. Mater. 2, 16028 (2016).

60. Cubuk, E. D., Schoenholz, S. S., Kaxiras, E. \& Liu, A. J. Structural properties of defects in glassy liquids. J. Phys. Chem. B 120, 6139-6146 (2016)

61. Ma, E. \& Ding, J. Tailoring structural inhomogeneities in metallic glasses to enable tensile ductility at room temperature. Mater. Today 19, 568-579 (2016).

62. Maloney, C. E. \& Lemaître, A. Amorphous systems in athermal, quasistatic shear. Phys. Rev. E 74, 16118 (2006).

63. Fawcett, T. An introduction to ROC analysis. Pattern Recognit. Lett. 27, 861-874 (2006).

64. Tharwat, A. Classification assessment methods. Appl. Comput. Informatics https://doi.org/10.1016/j.aci.2018.08.003 (2018).

65. Lechner, W. \& Dellago, C. Accurate determination of crystal structures based on averaged local bond order parameters. J. Chem. Phys. 129, 114707 (2008).

66. Plimpton, S. Fast parallel algorithms for short-range molecular dynamics. J. Comput. Phys. 117, 1-19 (1995).

\section{Acknowledgements}

The authors thank L.F. Zhang, J. Ding, E. Ma, and Z. Fan for beneficial discussions. A.J. acknowledges support from U.S. Department of Energy, Office of Basic Energy Sciences, Early Career Research Program, which intellectually led the effort. Q.W. also acknowledges the support of National Natural Science Foundation of China (51701190). This research used resources of the National Energy Research Scientific Computing Center (NERSC), U.S. Department of Energy Office of Science User Facility operated under Contract No. DE-AC02-05CH11231, and the National Supercomputing Center in Shenzhen, China.

\section{Author contributions}

Q.W. and A.J. developed the plan for this study. Q.W. designed the features, performed the simulations, and developed the machine learning models. Q.W. and A.J. analyzed the results and wrote the manuscript.

\section{Competing interests}

The authors declare no competing interests.

\section{Additional information}

Supplementary information is available for this paper at https://doi.org/10.1038/s41467019-13511-9.

Correspondence and requests for materials should be addressed to Q.W. or A.J.

Peer review information Nature Communications thanks Jan Schroers, Logan Ward, and the other, anonymous reviewer(s) for their contribution to the peer review of this work.

Reprints and permission information is available at http://www.nature.com/reprints

Publisher's note Springer Nature remains neutral with regard to jurisdictional claims in published maps and institutional affiliations.

cc (i) Open Access This article is licensed under a Creative Commons Attribution 4.0 International License, which permits use, sharing, adaptation, distribution and reproduction in any medium or format, as long as you give appropriate credit to the original author(s) and the source, provide a link to the Creative Commons license, and indicate if changes were made. The images or other third party material in this article are included in the article's Creative Commons license, unless indicated otherwise in a credit line to the material. If material is not included in the article's Creative Commons license and your intended use is not permitted by statutory regulation or exceeds the permitted use, you will need to obtain permission directly from the copyright holder. To view a copy of this license, visit http://creativecommons.org/ licenses/by/4.0/.

(C) The Author(s) 2019 